\title{
Internal Modes of Solitons and Near-Integrable Highly-Dispersive Nonlinear Systems
}

\author{
Oksana V. CHARKINA and Mikhail M. BOGDAN \\ B. Verkin Institute for Low Temperature Physics and Engineering of the NAS of Ukraine, \\ 47 Lenin Ave., Kharkiv, 61103 Ukraine \\ E-mail: charkina@ilt.kharkov.ua,bogdan@ilt.kharkov.ua
}

Received November 30, 2005, in final form April 11, 2006; Published online April 28, 2006

Original article is available at http://www.emis.de/journals/SIGMA/2006/Paper047/

\begin{abstract}
The transition from integrable to non-integrable highly-dispersive nonlinear models is investigated. The sine-Gordon and $\varphi^{4}$-equations with the additional fourth-order spatial and spatio-temporal derivatives, describing the higher dispersion, and with the terms originated from nonlinear interactions are studied. The exact static and moving topological kinks and soliton-complex solutions are obtained for a special choice of the equation parameters in the dispersive systems. The problem of spectra of linear excitations of the static kinks is solved completely for the case of the regularized equations with the spatiotemporal derivatives. The frequencies of the internal modes of the kink oscillations are found explicitly for the regularized sine-Gordon and $\varphi^{4}$-equations. The appearance of the first internal soliton mode is believed to be a criterion of the transition between integrable and non-integrable equations and it is considered as the sufficient condition for the nontrivial (inelastic) interactions of solitons in the systems.
\end{abstract}

Key words: solitons; integrable and non-integrable equations; internal modes; dispersion

2000 Mathematics Subject Classification: 34A05; 34A34; 35G25

We dedicate this paper to the memory of A.M. Kosevich.

\section{Introduction}

The soliton theory of completely integrable systems proposed a new basis of nonlinear fundamental excitations [1]. These elementary waves are solitons (antisolitons), breathers (solitonantisoliton bound states) and linear waves of the continuous spectrum. Any localized initial condition evolves as a superposition of such basic excitations. In the case of trivial (elastic) interaction between solitons the nonlinear excitations undergo only the phase and mass center shifts as a result of pair collisions. Then the initial profile is transformed asymptotically in the long time limit, in general, into a sequence of solitons and breathers existing upon linear wave background.

Soliton interactions can be described explicitly by the use of a multisoliton formula. To study interaction between a soliton and linear waves one needs to solve the equation linearized near the given soliton solution. It is remarkable that an exact solution of the problem can be also obtained from the multisoliton formula. For this purpose it is enough to make a special choice of parameters in the formula, which provides specification of the soliton and a near-zero amplitude limit for breather excitations [2].

In this paper we give a short survey of stability properties and linear excitation spectra of solitons in the sine-Gordon and $\varphi^{4}$-equations and their Boussinesq-like generalizations. Then we present a statement about spectrum of linear excitations of a soliton in a completely integrable 
system with trivial interaction between solitons. We justify the hypothesis which contends that presence of the internal oscillation mode in a soliton spectrum serves as a sufficient condition of non-integrability of a nonlinear system [3]. We use this criterion to state a crossover from the integrable sine-Gordon equation to its non-integrable generalizations with higher-order derivatives and dispersive nonlinear terms as well to conclude about non-integrability of corresponding $\varphi^{4}$-systems. These new equations possess exact moving kink or soliton complex solutions but they appear to be non-integrable.

\section{Kink excitation spectra in the sine-Gordon and $\varphi^{4}$-systems}

One of most important problems concerning the soliton dynamics in integrable and non-integrable systems is stability investigation. To solve the problem analytically one has to find a spectrum of linear excitations of the soliton. Usually this approach is demonstrated by consideration of the sine-Gordon equation (SGE) as an example:

$$
u_{t t}-u_{x x}+\sin u=0 .
$$

The well-known moving kink solution of the SGE has the following form:

$$
u_{s}=4 \arctan \left(\exp \frac{x-V t}{\sqrt{1-V^{2}}}\right) .
$$

Due to the Lorentz invariance of the SGE it is enough to determine a spectrum of kink excitations of a static solution $u_{s}$ with $V=0$. For the amplitude of small deviations from a kink profile

$$
u-u_{s}=\psi(x) \exp i \omega t
$$

it is easy to obtain the linear Schrödinger-type equation with the simplest reflectionless potential [4]:

$$
\left(-\frac{d^{2}}{d x^{2}}+1-\frac{2}{\cosh ^{2} x}\right) \psi(x)=\omega^{2} \psi(x) .
$$

Eigenfunctions of the equation correspond to the translational mode with the zero eigenfrequency

$$
\psi_{0}(x)=1 / \cosh x, \quad \omega_{0}=0
$$

and to waves of the continuous spectrum:

$$
\psi_{k}(x)=(\tanh x+i k) \exp i k x, \quad \omega_{k}=\sqrt{1+k^{2}} .
$$

No instability mode with $\omega^{2}<0$ exists in the spectrum hence the kink in the integrable SGsystem is stable. We note also that there is no additional localized mode with a discrete eigenvalue lying in the frequency gap $0<\omega<1$.

The another situation takes place in the non-integrable $\varphi^{4}$-equation:

$$
\varphi_{t t}-\varphi_{x x}-2\left(\varphi-\varphi^{3}\right)=0 .
$$

In this case there is also an exact kink solution:

$$
\varphi_{s}=\tanh \frac{x-V t}{\sqrt{1-V^{2}}} .
$$

Then for the amplitude $f(x)$ of small deviations from a static kink

$$
\varphi-\varphi_{s}=f(x) \exp i \omega t
$$


one finds the linear equation with one more reflectionless potential:

$$
\left(-\frac{d^{2}}{d x^{2}}+1-\frac{6}{\cosh ^{2} x}\right) f(x)=\omega^{2} f(x) .
$$

However, besides the translational mode with the zero frequency:

$$
f_{0}(x)=1 / \cosh ^{2} x, \quad \omega_{0}=0
$$

and continuum waves $f_{k}(x)$ with $\omega_{k}=\sqrt{4+k^{2}}$, the eigenfrequency spectrum of the equation contains the additional discrete eigenvalue corresponding to the internal mode of kink oscillations:

$$
f_{1}(x)=\frac{\sinh x}{\cosh ^{2} x}, \quad \omega_{1}=\sqrt{3} .
$$

Since all $\omega_{i}^{2} \geq 0$ then the kink of the $\varphi^{4}$-equation is also stable. But in contrast to the SG kink it possesses an intrinsic structure giving rise to an internal degree of freedom. The internal mode corresponds to a localized oscillation of an effective width of the kink.

Analogous situation takes place in the double sine-Gordon equation [5]

$$
u_{t t}-u_{x x}+\sin u \cos u+h \sin u=0,
$$

where the internal mode of the wobbler solution describes antiphase oscillations of two composite kinks. In this case the frequency of the internal mode becomes a function of the parameter $h$. Numerical simulations of the double sine-Gordon and $\varphi^{4}$-equations demonstrate effects of inelastic interactions between solitons, including resonant phenomena $[5,6]$. It is natural to suggest that the appearance of the internal mode in these models is connected with a non-integrability of the systems. This question is analyzed in the next section.

\section{Existence of internal mode as a non-integrability criterion}

The above consideration of soliton excitations allows to formulate the following statement: in a completely integrable system with only trivial (elastic) interactions between solitons a spectrum of linear excitations for a soliton can consist only of a translational (Goldstone) mode and continuum waves. Here and further we use the terminology of completely integrable systems, trivial and non-trivial interactions following those of V.E. Zakharov's papers (see, e.g., [7]). Well-known examples of the completely integrable systems with trivial soliton interactions are the sine-Gordon, nonlinear Schrödinger and Landau-Lifshitz equations. In such systems a basis of fundamental excitations consists of solitons, breathers and linear waves. To solve a spectral problem for the equations linearized near a soliton solution one can use a multisoliton formula. Then a soliton solution with arbitrary small perturbations can be presented by a combination of a soliton and breathers with near-zero amplitudes. In a small amplitude limit a breather is delocalized and transformed into a linear wave with a frequency belonging to a continuous spectrum. No linear localized mode corresponding to a discrete eigenvalue in the frequency gap can be obtained from this basis of fundamental excitations. Exception is the existence of a translational mode which follows from the translational invariance of the systems. Hence the spectrum of linear excitations of the soliton in the case of the trivial soliton interaction consists of the translational (Goldstone) mode and continuum waves. Thus it appears that solitons in a completely integrable system with only trivial interactions between solitons have no internal modes in their spectra of linear excitations. And vice versa, presence of the internal mode in a soliton spectrum should be considered as a sufficient condition at least for existence 
of non-trivial interaction between solitons in a nonlinear system. The last statement can be strengthened and formulated as the following conjecture.

Hypothesis. Presence of the internal mode in a spectrum of linear excitations of a soliton is a sufficient condition of the non-integrability of corresponding soliton-bearing nonlinear equations.

It should be noted that the absence of the internal mode in a linear excitation spectrum of a soliton is not a sufficient condition of the integrability of the soliton-bearing system. The main argument in favour of the hypothesis is that in the completely integrable system only the breather has its frequency in the gap between the zero and the continuous spectrum due to the finite value of its amplitude. Hence the existence of another type of nonlinear localized excitation with frequency in the gap would be assumed to obtain the internal mode oscillation as its linear limit. Such kind of soliton excitations is not known yet, at least for integrable systems.

The hypothesis first was formulated in the paper [3] and used for investigation of the transition from the integrable Landau-Lifshitz equation to a non-integrable one describing the biaxial ferromagnet placed in a magnetic field. Later this idea was used in the calculation of detachment of the internal mode from the continuous spectrum in the double sine-Gordon and near-discrete nonlinear Schrödinger equations [8].

\section{Soliton motion and instabilities in highly dispersive systems}

A soliton motion in the above examples of integrable equations does not influence the soliton stability. It is not a common case for integrable systems. There are known integrable systems with nontrivial interactions between solitons which exhibit instabilities in soliton dynamics [9, 10, 11]. The most famous example is the Boussinesq equation $[7,12,13,14]$ :

$$
u_{t t}-u_{x x}-6\left(u^{2}\right)_{x x}-u_{x x x x}=0 .
$$

It is easy to see that in this equation even the trivial solution $u=0$ is unstable with respect to short waves $u=a \exp (\gamma t) \cos k x$ of a continuous spectrum with wave numbers $k>1$. An exact analysis [9] of linear stability of a soliton of the Boussinesq equation also indicates the existence of an instability of the solution. Nevertheless it was shown that a nonlinear stage of the instability can be described by almost independent evolution of the growing mode [12] which destroys really neither solitons nor breathers [14].

However, there is another channel of the soliton instability directly connected with the nontrivial interaction between solitons in the Boussinesq equation with the opposite sign of the fourth-order derivative term [13]. Recently the Boussinesq equation was revisited and investigated in detail by the dressing method [7]. As a result of the consideration, a soliton decay into a pair of composite solitons (or its collapse) had been described explicitly. Linear stability analysis of the decaying soliton at the initial stage of this inelastic process has to lead to an existence of the instability mode [10] which corresponds to a localized eigenfunction. Here we should emphasize that we distinguish this instability mode corresponding to a growing solution from the internal mode which oscillates in time. To our knowledge, the internal mode has not been found yet in integrable equations with the non-trivial soliton interaction.

Taking into account higher-order derivatives to describe strong dispersion in the sine-Gordon and double sine-Gordon equations one comes to the highly dispersive non-integrable models [15]. These equations possess the soliton-complex solutions which can propagate with the fixed velocity. For example the dispersive sine-Gordon equation

$$
u_{t t}-u_{x x}-\beta u_{x x x x}+\sin u=0
$$


has the $4 \pi$-soliton complex solution in the form

$$
u_{c}=8 \arctan \left[\exp \varepsilon_{0}\left(x-V_{0} t\right)\right]
$$

where $\varepsilon_{0}$ and $V_{0}$ are definite functions of the dispersive parameter $\beta$. The soliton complex is a specific bound state of identical solitons, which is formed due to strong dispersion (see [15] for a survey). Note that a highly dispersive system is not integrable if this kind of the solution is revealed in the corresponding dispersive equations.

To avoid an instability with respect to short waves the regularized dispersive sine-Gordon equation with a mixed fourth-order derivative was introduced [15]:

$$
u_{t t}-u_{x x}-\beta u_{t t x x}+\sin u=0 .
$$

It also has the exact soliton-complex solution of the same form (1) but with different velocity dependence on the dispersive parameter $\beta$, namely:

$$
V_{r}=\sqrt{1+\frac{\beta}{3}}-\sqrt{\frac{\beta}{3}}
$$

Dynamical properties of the $2 \pi$-kink and the $4 \pi$-soliton complex in the regularized equation (2) have been studied in detail. In particular a spectrum of linear excitations of the static kink in the regularized sine-Gordon equation has been found exactly [16]. It was shown that, depending on the $\beta$ value, one or more internal modes are present in the spectrum. Additionally for the regularized dispersive $\varphi^{4}$-equation a complete spectrum of internal modes of kink oscillations was found exactly. Thus one of the advantages of the regularized dispersive equations is the fact that a process of the appearance of the internal modes of static kinks can be described explicitly.

It should be noted that there are several mechanisms of formation of multikinks and soliton bound states. At first it was found that interactions of oscillating soliton tails in dispersive media lead to the formation of bunches of solitons, consisting of two or more well-distinguishable humps $[17,18]$. Later it has been shown that solitons coexisting with resonant radiation can form bound states with purely solitonic asymptotics due to some kind of the radiation interference effect $[19,20]$. These multisoliton bound states have been called embedded solitons, implying their existence in the continuous spectrum.

In the paper [15] the concept of the soliton-complex in a nonlinear dispersive medium was proposed. It was shown that solitons in a strongly dispersive medium possess an internal structure and their interaction depends on intrinsic properties such as flexibility. Due to this dependence, the potential energy turns out to be a non-monotonic function of the distance between solitons. As a result, even identical topological solitons can attract each other and form a bound soliton complex which can move without any radiation in strongly dispersive media. These bound soliton states with zero and small distances between composite solitons have been called the soliton complexes. Their formation cannot be described by the soliton perturbation theory. Such soliton complexes appear even in systems with the effective strong dispersion, for example in nonlinear Schrödinger equation with the parametric pumping and dissipation [21]. The soliton complex in this case is formed due to neither oscillatory soliton tails interaction nor embedded soliton interaction but as a result of a strong multi-soliton interaction.

The regularized equations give a possibility to study soliton complexes in conditions when the influence of radiation is minimized. Indeed, in the equation (2) the continuous spectrum of linear waves degenerates into a single frequency $\omega_{0}=1$ for the parameter $\beta=1$. At the same time the number of kink internal modes become infinite and they play a principal role in soliton-complex dynamics. 
More than thirty years ago A. Kosevich and A. Kovalev combined the sine-Gordon and the modified Boussinesq equations to describe the crowdion (dislocation) motion in crystal with nonlinear interaction between nearest atoms [22]. They showed that the equation (KKE)

$$
u_{t t}-u_{x x}+\sin u-\gamma u_{x}^{2} u_{x x}-\beta u_{x x x x}=0
$$

has a moving crowdion solution with an arbitrary value of the velocity parameter $V$

$$
u_{c r}=4 \arctan [\exp \varepsilon(x-V t)]
$$

if the relation $\gamma=3 \beta / 2$ takes place. Here the dependence of the parameter $\varepsilon$ on the velocity is the following:

$$
\varepsilon=\sqrt{\frac{1-V^{2}}{2 \beta}\left(\sqrt{1+\frac{4 \beta}{\left(1-V^{2}\right)^{2}}}-1\right)} .
$$

Besides, for the equation, combining the Boussinesq and $\varphi^{4}$-equations,

$$
\varphi_{t t}-\varphi_{x x}-2\left(\varphi-\varphi^{3}\right)-\beta \varphi_{x x x x}+\alpha \varphi_{x} \varphi_{x x}=0
$$

authors showed the existence of a moving kink solution with a definite velocity which is a function of a special combination of parameters $\beta$ and $\alpha$.

A year later after the paper [22] K. Konno et al derived and solved by the inverse scattering method the equation [23]:

$$
u_{x t}-\sin u+\beta\left(u_{x x x x}+\frac{3}{2} u_{x}^{2} u_{x x}\right)=0 .
$$

This equation was reduced by the use of the Hirota transformation

$$
u=2 i \ln \frac{f^{*}}{f}
$$

to the following bilinear equations (see, e.g., [24])

$$
\begin{aligned}
& \left(D_{x} D_{t}+\beta D_{x}^{4}\right) f \cdot f=\frac{1}{2}\left(f^{2}-f^{* 2}\right), \\
& D_{x}^{2} f \cdot f^{*}=0,
\end{aligned}
$$

where Hirota operators are defined as follows [25]

$$
D_{x}^{m} D_{t}^{n} a \cdot b=\left.\left(\partial_{x}-\partial_{x^{\prime}}\right)^{m}\left(\partial_{t}-\partial_{t^{\prime}}\right)^{n} a(x, t) b\left(x^{\prime}, t^{\prime}\right)\right|_{x=x^{\prime}, t=t^{\prime}} .
$$

The bilinear equations (6) and (7) are solved exactly, resulting in the multisoliton solution.

In conclusion of the section we would like to note that only additional integrals of motion cannot guarantee, obviously, complete integrability of nonlinear equations. Here we mention, for example, the near-integrable dispersive equation by Leo et al [26]:

$$
u_{t}-u_{x} \sin u+\beta u_{x x x}=0 \text {, }
$$

which has three independent integrals of motion but the quasi-elastic soliton interaction in this equation is confirmed only numerically yet. 


\section{Kinks and soliton complexes in highly dispersive models with anharmonic interatomic interactions}

Instead of the use of a variational approach [27] for studying systems with anharmonic interatomic interactions, in this section we seek for exact solutions of the regularized versions of the dispersive Boussinesq-like sine-Gordon and $\varphi^{4}$-equations. However first of all we would like to conclude about the integrability of the Kosevich-Kovalev equation. For the case $\gamma=3 \beta / 2$ it has remained an open problem. There was a premature conclusion about its integrability [28], apparently based on similarity of this special case of the KKE and equation (4). We use the Hirota transformation (5) to reduce the KKE to a set of bilinear equations

$$
\begin{aligned}
& \left(D_{t}^{2}-D_{x}^{2}-\beta D_{x}^{4}\right) f \cdot f=\frac{1}{2}\left(f^{2}-f^{* 2}\right), \\
& D_{x}^{2} f \cdot f^{*}=0 .
\end{aligned}
$$

It is easy to verify that these equations do not have a multisoliton solution in contrast to equations (6) and (7). Nevertheless in the case $\gamma \neq 3 \beta / 2$ we find the exact soliton-complex solution of the KKE:

$$
u_{c}=8 \arctan [\exp \varepsilon(x-V t)], \quad \varepsilon^{4}=\frac{1}{3 \beta-8 \gamma}, \quad V=\sqrt{1-2 \frac{\beta-4 \gamma}{\sqrt{3 \beta-8 \gamma}}} .
$$

Evidently the solution exists when $\beta>8 \gamma / 3$ and the velocity $V<1$ if $\beta>4 \gamma$.

Now we consider the regularized dispersive sine-Gordon equation with a nonlinear dispersive term:

$$
u_{t t}-u_{x x}+\sin u-\gamma u_{x}^{2} u_{x x}-\beta u_{t t x x}=0 .
$$

At first we show that this equation has a static kink solution for arbitrary values of parameters $\gamma$ and $\beta$. In fact after one integration of the equation (8) we obtain for $u(x)$

$$
d u / d x=\left[\left(\sqrt{1+8 \gamma \sin ^{2}(u / 2)}-1\right) / \gamma\right]^{1 / 2} .
$$

The $2 \pi$-kink solution exists for any positive $\gamma$ and at small values of the parameter we find out the expansion

$$
u_{k}(x) \approx 4 \arctan (\exp (x))-2 \gamma \frac{\sinh (x)}{\cosh ^{2}(x)}
$$

With increasing $\gamma$ the effective kink width also increases. We have analyzed a spectral problem of the equation linearized near the kink solution (9). By the use of the perturbation theory with a small parameter $\gamma$ we have revealed detachment of the internal mode from the continuous spectrum. We have found that a difference of the internal mode frequency from unity is proportional to the first power of the parameter $\gamma$. Thus we conclude that the equation (8) is not integrable.

Besides the static kink the equation (8) possesses the soliton-complex solution

$$
u_{c}=8 \arctan [\exp \kappa(x-V t)],
$$

where parameters $\kappa$ and $V$ obey the following equations

$$
\kappa^{4}=\frac{1}{3 \beta V^{2}-8 \gamma}, \quad V^{2}=\frac{\kappa^{2}-1}{\kappa^{2}\left(1-\beta \kappa^{2}\right)} .
$$


The latter equations determine fixed velocity and effective width of the soliton complex. Although there is the lower boundary for admissible values of the velocity $(V>\sqrt{8 \gamma / 3 \beta})$ nevertheless at small enough $\gamma$ there is a definite range of $\beta$-values which provides valid quantities of parameters $\kappa$ and $V$. For example, for $\beta=0.03$ and $\gamma=0.025$ values of the velocity $V_{0}$ and the parameter $\kappa_{0}$ are equal to 0.913 and 1.47 respectively.

We propose one more regularized version of the dispersive Boussinesq-like sine-Gordon equation

$$
u_{t t}-u_{x x}+\sin u-\gamma u_{x}^{2} u_{t t}-\beta u_{t t x x}=0 .
$$

The equation possesses the moving kink and soliton complex solutions which are very similar in their forms to corresponding solutions of the KKE. In the case $\gamma=3 \beta / 2$ there exists the $2 \pi$-kink propagating with an arbitrary velocity less than unity:

$$
u_{s}=4 \arctan [\exp \varepsilon(x-V t)], \quad \varepsilon=\sqrt{\frac{1-V^{2}}{2 \beta V^{2}}\left(\sqrt{1+\frac{4 \beta V^{2}}{\left(1-V^{2}\right)^{2}}}-1\right)} .
$$

Such moving solution does not exist in the case of $\gamma \neq 3 \beta / 2$. However a static $2 \pi$-kink exists for arbitrary values of $\beta$ and $\gamma$ :

$$
u_{k}=4 \arctan (\exp (x)) .
$$

The kink exhibits an intrinsic structure which manifests itself in its internal dynamics and complex soliton interactions as it is shown below.

For values $\gamma \neq 3 \beta / 2$ the $4 \pi$-soliton complex solution appears:

$$
\begin{aligned}
& u_{c}=8 \arctan [\exp \varepsilon(x-V t)], \\
& V=\frac{\sigma}{\sqrt{1+\sigma^{2}}+1}, \quad \varepsilon=\sqrt{\frac{\sqrt{1+\sigma^{2}}+1}{\sigma \sqrt{3 \beta-8 \gamma}}},
\end{aligned}
$$

where

$$
\sigma=\frac{\sqrt{3 \beta-8 \gamma}}{\beta-4 \gamma}, \quad \beta>4 \gamma .
$$

It should be noted that this solution exists also for $\beta=1$ when the continuous spectrum degenerates and it is interesting to investigate numerically the soliton-complex stability properties in this case.

Here we present results of numerical simulations of the equation (10) for the soliton-complex dynamics when the parameter $\beta=1$ and $\gamma$ is very small. If one starts at $t_{0}=0$ with the soliton-complex profile which has the initial velocity $V_{\text {in }}=0.4$ less than $V_{r}$ then the result of simulation will be as shown in Fig. 1. The profile evolves with throwing down the superfluous energy by exciting the internal mode, and it dissociates finally into two moving kinks.

The soliton complex given by the exact solution (12) appears to be stable and moves radiationlessly in the dispersive media.

In conclusion of this section we present the regularized version of the generalized dispersive $\varphi^{4}$-equation (3) of the following form:

$$
\varphi_{t t}-\varphi_{x x}-2\left(\varphi-\varphi^{3}\right)-\beta \varphi_{x x t t}+\alpha \varphi_{x} \varphi_{t t}=0 .
$$

Besides the moving kink which is analogous to the solution of the equation (3) there exists an exact static kink solution for arbitrary parameters $\beta$ and $\alpha$ :

$$
\varphi_{s}(x)=\tanh (x) .
$$

In the next section explicit expressions for frequencies of internal modes of static kinks are obtained for the regularized dispersive sine-Gordon and $\varphi^{4}$-equations. 


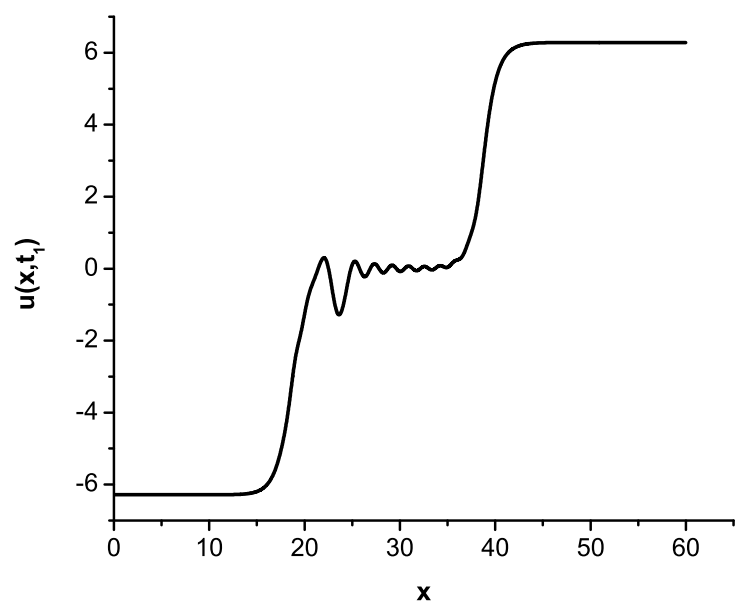

Figure 1. Dissociation of the soliton complex profile with the initial velocity $V_{\text {in }}=0.4$ in the dispersive sine-Gordon equation (10) with $\beta=1$ and $\gamma=0.025$. The numerical solution profile is shown at the moment $t_{1}=60$.

\section{Internal modes of kinks in the dispersive sine-Gordon and $\varphi^{4}$ equations}

A remarkable property of the regularized equation (10) is that the problem of the spectrum of internal modes of a static kink can be solved exactly.

Let us seek for a solution of equation (10) in the form:

$$
u=u_{k}+\psi(x) \exp i \omega t
$$

where $u_{k}$ is given by equation $(11)$ and $\psi(x) \ll u_{k}$. Then the linearized equation for function $\psi(x)$ is obtained as follows:

$$
\left[-\left(1-\beta \omega^{2}\right) \frac{d^{2}}{d x^{2}}+1-\frac{2}{\cosh ^{2} x}\left(1-2 \gamma \omega^{2}\right)\right] \psi=\omega^{2} \psi
$$

Discrete set of eigenvalues is given by the equation (see, e.g., [4]):

$$
\sqrt{\frac{1}{4}+\frac{2\left(1-2 \gamma \omega^{2}\right)}{1-\beta \omega^{2}}}-\sqrt{\frac{1-\omega^{2}}{1-\beta \omega^{2}}}=n+\frac{1}{2},
$$

where $n$ is an integer. The zero eigenvalue corresponds to the translational mode and $n=0$. The number of further levels, i.e. the number of internal modes, is determined by values of the parameters $\beta$ and $\gamma$.

Let us consider some special cases of the equation (14):

1. $\gamma=0$. For this case the dispersive SGE was studied in the paper [16]:

$$
\sqrt{\frac{1}{4}+\frac{2}{1-\beta \omega^{2}}}-\sqrt{\frac{1-\omega^{2}}{1-\beta \omega^{2}}}=n+\frac{1}{2} .
$$

Depending on the dispersive parameter $\beta$, internal modes detach from the continuous spectrum at the following values of the dispersive parameter $\beta$ :

$$
\beta_{n}=1-\frac{2}{n(n+1)} .
$$


The first mode frequency is presented explicitly as follows:

$$
\omega_{1}=\sqrt{\frac{1}{\beta} \frac{\Delta^{2}-9}{\Delta^{2}-1}}, \quad \Delta(\beta)=\frac{6 \beta+\sqrt{17 \beta^{2}-10 \beta+9}}{1+\beta} .
$$

Analysis of the expansion of $\omega_{1}(\beta)$ at small $\beta$ indicates clearly the appearance of the first internal mode as soon as $\beta \neq 0$ and hence loss of the integrability of this variant of the sine-Gordon equation.

It should be noted that in the case of the continuous spectrum degeneration $(\beta=1)$ all the infinite set of the frequencies of internal modes is expressed very simply:

$$
\omega_{n}=\sqrt{1-\frac{2}{(n+1)(n+2)}} .
$$

In this limit the dynamics of soliton complexes depends strongly on possibility of excitation of the internal modes of composite kinks. This circumstance leads to a large variety of resonant phenomena in soliton interactions in the dispersive equation (10).

2. $\gamma=\beta / 2$. It is easy to be convinced that this is the case of a reflectionless potential in the equation (13)

$$
\sqrt{\frac{1-\omega^{2}}{1-\beta \omega^{2}}}=1-n
$$

It is evident that there are only two discrete eigenvalues: (i) values $n=0$ and $\omega=0$ correspond to the translational mode; (ii) values $n=1$ and $\omega=1$ correspond to the edge of the continuous spectrum. Such kind of the spectrum is typical for integrable systems. Unfortunately the absence of internal mode cannot serve as the sufficient condition of the integrability. Therefore we doubt complete integrability of the equation in this case but could expect the near-integrable behavior of soliton dynamics.

3. $\gamma=3 \beta / 2$. This is the case when moving kink exists. For the static kink limit the discrete eigenvalue equation is the following

$$
\sqrt{\frac{1}{4}+\frac{2\left(1-3 \beta \omega^{2}\right)}{1-\beta \omega^{2}}}-\sqrt{\frac{1-\omega^{2}}{1-\beta \omega^{2}}}=n+\frac{1}{2} .
$$

Here also no internal mode exists between the zero frequency and a continuous spectrum. However the potential well in the equation (13) appears to be not reflectionless as usually in integrable systems. The last argument works rather against the suggestion about the equation integrability in this case.

Spectrum of internal modes of a static $\varphi^{4}$-kink is given by the equation:

$$
\sqrt{\frac{1}{4}+\frac{6+\alpha \omega^{2}}{1-\beta \omega^{2}}}-\sqrt{\frac{4-\omega^{2}}{1-\beta \omega^{2}}}=n+\frac{1}{2} .
$$

This equation has discrete set of solutions for eigenfrequencies of internal modes for any positive $\alpha$ and $0 \leq \beta<1$. A structure of the discrete spectrum of internal modes is similar to that of a kink of the dispersive $\varphi^{4}$-equation [16]. In the latter paper the detailed study of the kink excitations spectrum for the case of $\alpha=0$ was performed.

Thus the analysis of presence of internal modes in the kink excitation spectrum justifies the fact of the non-integrability of the corresponding dispersive sine-Gordon and $\varphi^{4}$-equations. Evidently, the internal structure of solitons in highly dispersive media can manifest itself in inelastic processes of the soliton interaction. Formation of the soliton complexes is one of consequences of such an interaction. Hence the existence of the soliton complex solution can be also considered as the argument in favour of the non-integrability of nonlinear equations. 


\section{Conclusions}

The results of the present consideration are summarized as follows:

1. Exact static and moving solutions describing kinks and soliton complexes are found in the equations describing highly dispersive models with nonlinear interatomic interactions. However the presence of the exact solutions (especially, moving kinks with arbitrary velocities) does not argue for complete integrability of the systems. In particular, it is demonstrated that the Kosevich-Kovalev equation is not completely integrable in the case $3 \gamma>8 \beta$ due to an existence of the soliton complex solution. It is also shown that there is not the standard multisoliton formula for this equation in the special case $\gamma=3 \beta / 2$.

2. As a result of application of the criterion of the non-integrability, based on presence of the internal mode in a soliton spectrum, it is shown that the regularized dispersive sineGordon equation with the fourth-order mixed derivative and the nonlinear dispersive term is not completely integrable for the $\gamma<\beta / 2$. Soliton dynamics in this system demonstrates a complex behavior including bound state formation and dissociation processes. However it should be expected for the case $\gamma=\beta / 2$ that soliton interactions in the system could be close with the dynamical behavior in the integrable model.

3. In general, finding of the exact solutions for spectra of linear excitations of solitons, including the internal modes, for the generalized sine-Gordon and $\varphi^{4}$-models allow us to make conclusions about the near-integrable soliton properties or a complex internal structure and the non-trivial dynamics in the highly dispersive systems. Finally it is believed that existence of the internal mode can serve as the criterion of non-integrability of a nonlinear system, supplementing efficiently other integrability checks, like, e.g., the Painlevé test.

[1] Ablowitz M.J., Segur H., Solitons and the inverse scattering transform, Philadelphia, SIAM, 1981.

[2] Dashen R., Hasslacher B., Neveu A., Particle spectrum in model field theories from semiclassical functional integral techniques, Phys. Rev. D, 1975, V.11, N 12, 3424-3450.

[3] Bogdan M.M., Kosevich A.M., Voronov V.P., Generation of the internal oscillation of soliton in a onedimensional non-integrable system, in Proceedings of IVth International Workshop "Solitons and Applications" (August 24-26, 1989, Dubna, USSR), Editors V.G. Makhankov, V.K. Fedyanin and O.K. Pashaev, Singapore, World Scientific, 1990, Part IV, 397-401.

[4] Landau L.D., Lifshitz E.M., Quantum mechanics, New York, Pergamon, 1977.

[5] Campbell D.K., Peyrard M., Sodano P., Kink-antikink interactions in the double sine-Gordon equation, Phys. D, 1986, V.19, N 2, 165-205.

[6] Campbell D.K., Schonfeld J.F., Wingate C.A., Resonance structure in kink-antikink interactions in $\phi^{4}$ theory, Phys. D, 1983, V.9, N 1-2, 1-32.

[7] Bogdanov L.V., Zakharov V.E., The Boussinesq equation revisited, Phys. D, 2002, V.165, N 3-4, $137-162$.

[8] Kivshar Yu.S., Pelinovsky D.E., Cretegny T., Peyrard M., Internal modes of solitary waves, Phys. Rev. Lett., 1998, V.80, N 23, 5032-5035.

[9] Berryman J.G., Stability of solitary waves in shallow water, Phys. Fluids, 1976, V.19, N 6, 771-777.

[10] Fal'kovich G.E., Spector M.D., Turitsyn S.K., Destruction of stationary solutions and collapse in the nonlinear string equation, Phys. Lett. A, 1983, V.99, N 6-7, 271-274.

[11] Kuznetsov E.A., Spector M.D., Fal'kovich G.E., On the stability of nonlinear waves in integrable models, Phys. D, 1984, V.10, N 3, 379-386.

[12] Yajima N., On a growing mode of the Boussinesq equation, Progr. Theoret. Phys., 1983, V.69, N 2, 678-680.

[13] Flytzanis N., Pnevmatikos S., Remoissenet M., Soliton resonances in atomic nonlinear systems, Phys. D, 1987, V.26, N 1-3, 311-320.

[14] Tajiri M., Murakami Y., On breather solutions to the Boussinesq equation, J. Phys. Soc. Japan, 1989, V.58, N 10, 3585-3590.

[15] Bogdan M.M., Kosevich A.M., Maugin G.A., Soliton complex dynamics in strongly dispersive medium, Wave Motion, 2001, V.34, N 1, 1-26, patt-sol/9902009. 
[16] Charkina O.V., Bogdan M.M., Internal modes of oscillations of topological solitons in highly dispersive media, Uzhgorod Univ. Sci. Herald. Series Physics, 2005, N 17, 30-37 (in Russian).

[17] Gorshkov K.A., Ostrovsky L.A., Interaction of solitons in nonintegrable systems: direct perturbation method and applications, Phys. D, 1981, V.3, N 3, 428-438.

[18] Kawahara T., Toh S., Pulse interactions in an unstable dissipative-dispersive nonlinear system, Phys. Fluids, 1988, V.3, N 8, 2103-2111.

[19] Champneys A.R., Malomed B.A., Yang J., Kaup D.J., Embedded solitons: solitary waves in resonance with the linear spectrum, Phys. D, 2001, V.152-153, 340-354, nlin.PS/0005056.

[20] Kolossovski K., Champneys A.R., Buryak A.V., Sammut R.A., Multi-pulse embedded solitons as bound states of quasi-solitons, Phys. D, 2002, V.171, N 3, 153-177.

[21] Barashenkov I.V., Zemlyanaya E.V., Stable complexes of parametrically driven damped nonlinear Schrödinger solitons, Phys. Rev. Lett., 1999, V.83, N 13, 2568-2571.

[22] Kosevich A.M., Kovalev A.S., The supersonic motion of a crowdion. The one-dimensional model with nonlinear interaction between the nearest neighbours, Sol. State Comm., 1973, V.12, N 8, 763-765.

[23] Konno K., Kameyama W., Sanuki H., Effect of weak dislocation potential on nonlinear wave propagation in anharmonic crystal, J. Phys. Soc. Japan, 1974, V.37, N 1, 171-176.

[24] Chen D.Y., Zhang D.J., Deng S.F., The novel multi-soliton solutions of the MKdV-sine Gordon equations, J. Phys. Soc. Japan, 2002, V.71, N 2, 658-659.

[25] Hirota R., Direct method of finding exact solutions of non-linear evolution equations, in Bäcklund Transformations, Editors A. Dold and B. Eckmann, Lecture Notes in Mathematics, Vol. 515, New York, SpringerVerlag, 1980, 40-68.

[26] Leo M., Leo R.A., Soliani G., Soliton-like solutions for a dispersive nonlinear wave equation, Progr. Theoret. Phys., 1978, V.60, N 1, 100-111.

[27] Braun O.M., Zhang Fei, Kivshar Yu.S., Vazquez L., Kinks in the Klein-Gordon model with anharmonic interatomic interactions: a variational approach, Phys. Lett. A, 1991, V.157, N 4-5, 241-245.

[28] Gendelman O.V., Manevich L.I., Exact soliton-like solutions in generalised dynamical models of quasi-onedimensional crystal, Zh. Eksper. Teoret. Fiz., 1997, V.112, N 4 (10), 1510-1515. 